\title{
The Influence of Organizational Commitment, Competence, Academic Atmosphere on Motivation and Performance (Study on State Elementary School Teachers in Region 3T: Lagging, Frontier, Outermost)
}

\author{
Alimudin Muhammad ${ }^{1 *}$, Muhammad Basir-Cyio², Bakri Hasanuddin ${ }^{3}$ \\ ${ }^{1.3}$ Tadulako University, Faculty of Economics and Business, Palu-Central Sulawesi, Indonesia \\ ${ }^{2}$ Tadulako University, Faculty of Agriculture, Palu-Central Sulawesi, Indonesia \\ *Corresponding Author
}

\begin{abstract}
The purpose of this study is to empirically test and explain (1) the effect of organizational commitment on motivation, (2) the effect of Competence on motivation, (3) the influence of the academic atmosphere on motivation, (4) the effect of organizational commitment on performance, (5) the effect of Competence on performance, (6) the effect of the academic atmosphere on performance, and (7) the effect of motivation on performance. This type of research is explanatory research. The research sample is teachers who work in the $3 \mathrm{~T}$ region (Lagging, Frontier, Outermost) in Tojo Una-Una Regency, Central Sulawesi Province, and the number of samples is 200 teachers. The data analysis method uses Structural Equation Modeling (SEM), the results show that (1) organizational commitment has a significant effect on motivation, (2) competence has no significant effect on,
\end{abstract}

Keywords: Organizational Commitment, Competence, Academic Atmosphere, Motivation, and Performance.

\section{INTRODUCTION}

$\mathrm{T}$ he development of an organization, both government and private, can be measured by the extent to which each individual achieves the performance. According toSusanto (2014), Performance can be understood as an achievement, result, or ability that is earned or shown in the implementation of work, obligations or tasks individuals, including teachers.

Tojo Una-Una Regency has been designated as a 3T area through the Decree of the Minister of Education and Culture of the Republic of Indonesia Number 123/P/2012 concerning Designation of Special Regions, spread over 8 sub-districts. The number of schools included in the disadvantaged category is 47 schools with 3,928 students. Characteristics of the $3 \mathrm{~T}$ region that are subject to limitations, the teachers who work in that area must have a high commitment to the organization. According to Allen and Mayer (2009) that organizational commitment consists of: three, namely: (1) affective commitment, (2) normative commitment, and (3) continuance commitment.
The importance of teacher commitment has attracted the attention of researchers, including Shadma and Rasmita, (2009), Tuğrul Mart, (2013), and Basilius and Anak Agung (2017). The results show that committed teachers tend to play their roles effectively what their jobs require and to build good teacher-student relationships consistent with professional values. The findings of Shadma and Rasmita (2009), Tuğrul Mart (2013), and Basilius and Anak Agung (2017) are reinforced by Suharto, et al. (2018); the results of the study explain that commitment directly affects the job performance of Civil Servants.

In addition to the teacher's commitments most crucial thing to note is the Competence of the teacher. Competence is seen from the education of elementary school teachers in the 3T region, the dominant education is Strata D2, and a small part has an undergraduate education. How vital is Competence, as explained bySpencer and Spencer (1993), that Competence is a characteristic that stands out for a person, ways of behaving, and thinking in all situations that last for an extended period? Five characteristics of Competence, according to Spencer and Spencer (1993), are: (1) Motive, is something that is thought and desired, (2) Traits, are physical characteristics and consistent responses to situations, (3) Selfconcept, namely attitudes, values, and the image of a person, (4) Knowledge, namely the information a person has in a particular field, and (5) Skills, namely the ability to perform tasks related to physical and mental. Thus, it can be concluded that Competence is needed as an essential characteristic for someone in doing work, by combining education,

Research on Competence about performance has been carried out by previous researchers, including Maende and Luvai (2014), Bambang et al. (2016), Haniatin (2016), and Sumual et al. (2017), Sammy et al. (2018). The findings reveal that professional teacher training, teacher discipline, and teacher competence positively and significantly affect 
performance. And the most significant influence contribution to performance is professional teacher training.

Characteristics of the $3 \mathrm{~T}$ region with all the limited facilities and infrastructure, teachers are required to have high commitment and good Competence, and teachers are also expected to create an academic atmosphere. According to Kurniawan (2013), the academic atmosphere is an ideal academic atmosphere that can be described by various activities (activities) in the school environment, which are characterized by harmonious interactions between teachersstudents, students, and teachers based on academic values. This is in line with the opinion of Bosque and Dore (1998) that the learning and teaching environment must implement six (6) functions, namely: (1) information, (2) communicating, (3) collaborating, (4) product, (5) buffer and (6) manage.

Regarding the academic atmosphere has attracted the attention of researchers, including Sarwat and Muhammad (2014), Shamaki and Timothy (2015), Ahmad et al. (2018), the results of descriptive research show the average value of the academic environment is 3.79 (good category). The indicator that gives the highest contribution to the intellectual atmosphere is: collaboration, and the lowest indicator is: instructional innovation. Thus, it can be concluded that the academic atmosphere or academic atmosphere can trigger teacher work motivation.

Furthermore, the government's attention to motivating teachers to stay in the $3 \mathrm{~T}$ region is through providing special allowances for teachers. The funding comes from the APBN and APBD. The form of material motivation is the essential driving force or stimulant that causes people to do something. This motivation is the guiding force that moves people to behave to achieve specific goals. According to Gibson et al. (2011), motivation is a force that can encourage him to do something that causes and directs behavior. Maslow (Kreitner and Kinicki; 2014) introduced a theory of motivation based on a hierarchy of needs, five basic needs that influence behavior- physiological, security, social, esteem, and self-actualization. In line with the opinion of Robbins and Timothy (2018), motivation describes the strength, direction, and persistence of a person to achieve goals.

How important teacher motivation is, has received serious attention from several researchers, including those conducted by Atiya and Palwasha (2016), Rhini et al. (2017), and Elizabeth (2019). The results of the study conclude that teacher motivation has a significant influence on performance. This study recommends that teachers should be given better explanations in terms of salary and promotion. Other research conducted by: research results show that: In addition, analysis by Hamidi et al. (2017) leads that (1) intrinsic factors that motivate teachers to persist in teaching in remote areas (a sense of responsibility for education, teachers enjoy work, teachers gain good appreciation from the local community,

\section{LITERATURE REVIEW}

\section{Relationship between Organizational Commitment and} Motivation

Previous researchers have carried out an empirical study of the relationship between organizational commitment and motivation. Empirical evidence shows that (a) organizational commitment has a positive and significant effect on work motivation, and (b) organizational commitment has a substantial contribution or influence of $76 \%$ on the variance of work motivation variables (Farida et al., 2016). Other empirical research conducted by Bano et al. (2019) and Tugrul (2013) explains that very high organizational commitment can lead to increased performance and efficiency of an employee in completing organizational tasks and goals. It can be demonstrated that organizational commitment can improve one's work motivation to better perform according to individual and organizational expectations.

H1: Organizational commitment has a significant effect on motivation Relationship of Competence with Motivation,

Robbins and Judge (2015) explain that Competence is "the ability or capacity of a person to do various tasks in a job, where this ability is determined by 2 (two) factors, namely intellectual ability and physical ability. Likewise, according to Veithzal et al. (2015), Competence is a fundamental characteristic possessed by a person that directly affects or can predict good performance. Because of the importance of competence in motivation, it has been the concern of previous researchers, among others, by Heriyanto et al. (2018), Utoyo et al., 2019). The findings state that Competence has a direct impact on work motivation. Based on previous empirical studies and studying the theories that have been described previously, the following hypotheses are formulated:

H2: Competence has a significant effect on motivation Relationship between Academic Atmosphere and Motivation

The relationship between the academic atmosphere and employee work motivation has become an exciting issue for previous researchers, including those conducted by Srikanthan and Dalrymple (2003), Thrash (2009), Siddique et al. (2011); Alafy et al. (2013), explained that the academic atmosphere is a learning process that adheres to a system of academic freedom and can form a campus climate that is ethical and moral. Likewise, Agustina's opinion, at al (2016) explains that the academic atmosphere is a condition that must be created to make the learning process run according to the vision, mission, and goals. In addition, Ahmad et al. (2018) research concluded that the academic atmosphere significantly affected motivation.

H3: Academic Atmosphere has a significant effect on motivation Relationship between Organizational Commitment and Performance

Previous research has proven that commitment has a significant influence on performance, among others, carried 
out by Anwar et al. (2019), this study reveals that Competence, motivation Jafri and Lhamo (2013) explains that employee organizational commitment is one of the attitudes that can lead to high performance. Employees committed to their organization are more likely to be better off than employees who are less committed because they put more effort on behalf of the organization towards its success and strive to achieve its goals and mission. Next, pThe research of Anwar et al. (2019) has proven that commitment has a significant effect on performance; a decrease in organizational commitment among teachers can affect the quality and quantity of their performance. Based on empirical studies and studying the theories that have been described previously, the following hypotheses are formulated:

Based on empirical studies and studying the theories that have been described previously, the following hypotheses are formulated:

H4: Organizational Commitment has a significant effect on performance Relationship between Competence and Performance

Competency research on performance has become the attention of previous researchers, among others, has been carried out by Bambang et al. (2016), Haniatin (2016) has proven empirically Competence has a significant effect on performance, especially in the development of teacher competencies, primarily academic and professional competencies. In addition, research conducted by Sammy et al. (2019), the results of his study recommend that teachers are encouraged to develop their competencies for effective delivery in the classroom. Thus, the research has proven that the competencies possessed will improve employee performance, including SDN teachers who work in the $3 \mathrm{~T}$ area. Based on empirical studies and studying the theory described previously, the following hypothesis is formulated:

H5: Competence has a significant effect on performance Relationship between Academic Atmosphere and Performance

Research on the academic atmosphere about performance has been the concern of previous researchers, among others, has been carried out by Sarwat and Muhammad (2014), where the results of the study explain that Robbins and Judge (2015) explain employee performance is a function of the interaction between ability and motivation. In line with Gibson et al. (2011), every employee in the organization is required to make a positive contribution through good performance, considering that organizational performance depends on the performance of its employees. Likewise, Simamora (2012) explains that performance is the level of work achievement efficiently and effectively, or employee performance is work performance. Thus, it can be concluded that the creation of an academic atmosphere will affect the overall performance in the process of achieving academic achievement.

Research on the academic atmosphere about performance has been the concern of previous researchers, among others, has been carried out by Sarwat and Muhammad (2014). The results of the study explain that a supportive school environment affects performance. In addition, research conducted by Raziq and Raheela (2015) and Hafeez et al. (2019) has been able to show that the academic atmosphere (workplace environment) has a significant influence on employee performance. Based on empirical studies and studying the theories that have been described previously, the following hypotheses are formulated:

H6: Academic Atmosphere has a significant effect on The Relationship between Motivation and Performance

\section{The Relationship between Motivation and Performance}

Motivation theory divides 3 basic needs in work motivation: achievement, power, and affiliation (Robbins and Judge, 2015). McClelland argued that individuals have potential energy reserves. How this energy is released and developed depends on the strength or motivation of the individual and the available situations and opportunities. McClelland describes three types of motivation, namely: (1) The need for achievement (N-Ach), (2) The need for power (Need for Power: N-Pow), and (3) the Need for Affiliation or friendship (Need for Affiliation: $N$-Aff). The relationship of motivation to performance has been carried out by Fei and Crispina (2019), Elizabeth (2019), and Rhini et al. (2017); his findings confirm that motivation affects the main construction to improve performance. This finding is different from the findings of Nawangsih and Linayaningsih (2015), where the study results show no significant relationship between motivation and performance.

Based on empirical studies and studying the theories that have been described previously, the following hypotheses are formulated:

\section{H7: Motivation has a significant effect on performance.}


The conceptual framework

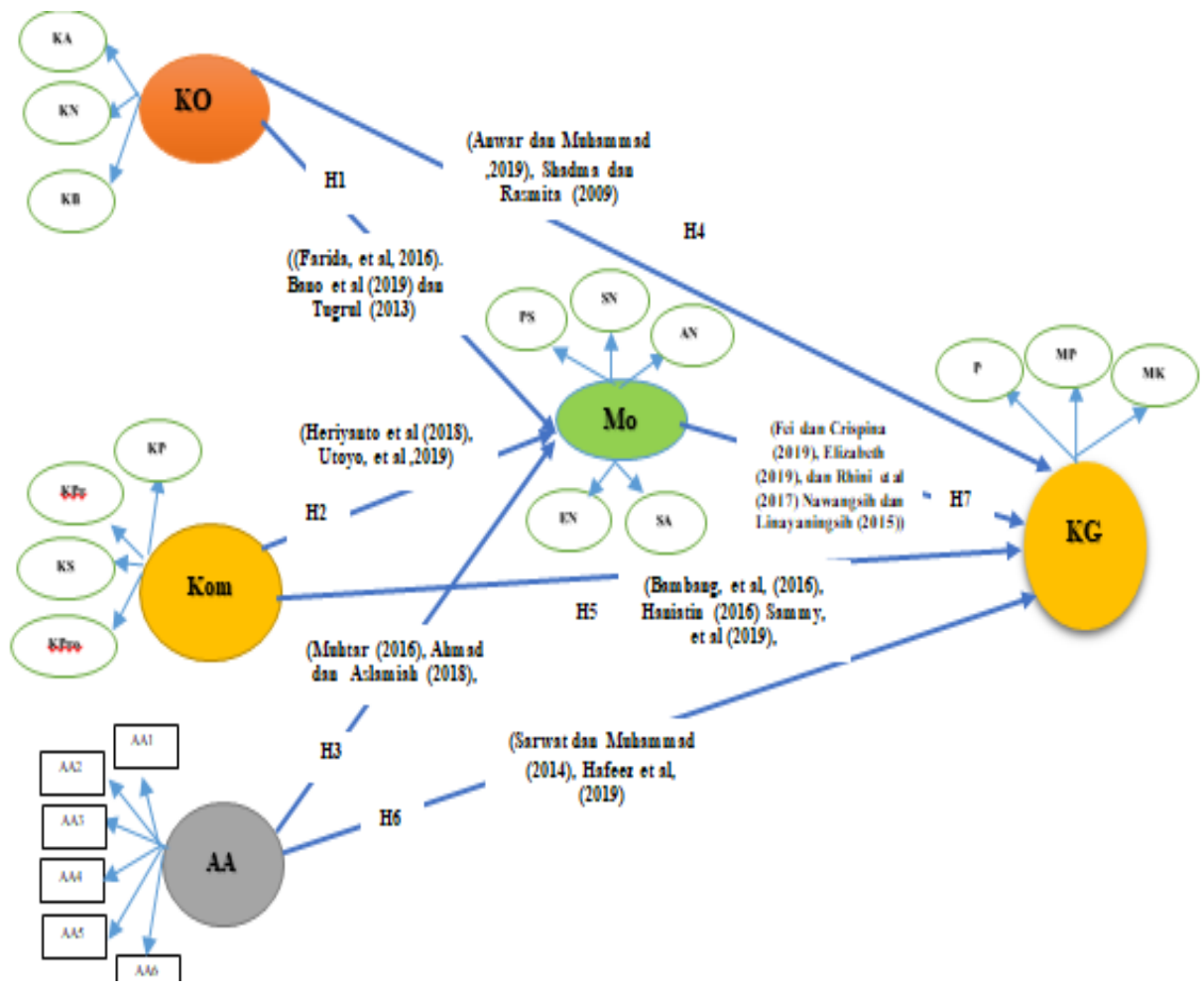

Figure 1: Research Structure Model

Information:

KO : Organizational Commitment Mo: Motivation

Kom : KAA competency: Academic Atmosphere

KG : Teacher Performance

\section{METHODOLOGY}

This type of research includes the explanatory kind of research. Explanatory is a type of research that highlights the relationship between research variables and tests hypotheses that have been formulated previously (Sekaran \& Bougie, 2017). The same opinion was expressed by Sugiyono (2017) and Sukmadinata (2009) that explanatory research is research that explains the position between the variables studied and the relationship between one variable and another. Furthermore, Sekaran \& Bougie (2017), when viewed from the research objective, this research is in the form of a descriptive and explanatory study through hypothesis testing.

\section{Sampling and data collection}

The population in this study were all SDN teachers left behind in the 3T region, as many as 396 teachers (250 PNS teachers and 146 non-PNS teachers). The sample size refers to the opinion of Ferdinand (2000) that the sample size for model testing using SEM is between 100-200 samples or depending on the number of parameters used in all latent variables, namely the number of parameters multiplied by 5 to 10 . In general, the structural equation model has at least 200 observations (Kelloway, 1998; Bacharuddin and Harapan, 2003). Furthermore, it is generally acceptable for a sample of 200 as a representative sample in SEM analysis. On the other hand, Kusnendi (2005) opinion states that the minimum sample size for SEM analysis is 100-200. Based on the opinion reference above, the researchers set 200 samples. The analysis carried out for the structural measurement model is using AMOS software release 20.0.

Table 1 below is a suitability index and cut-off value to test whether a model can be accepted or rejected according to Ferdinand (2000); the CFI criteria can be presented as follows.

Table 1. CFI (Comparative Fit Index)

\begin{tabular}{|c|c|c|}
\hline No & The goodness of Fit Index & \begin{tabular}{c} 
Cut off value \\
\hline 1
\end{tabular} \\
\hline 2 & Chi-Square & $\begin{array}{c}\text { Expected to be small below the table } \\
\text { value }\end{array}$ \\
\hline 3 & Significance & $>0.05$ \\
\hline 4 & RMSEA & $<0.08$ \\
\hline 5 & GFI & $>0.90$ \\
\hline 6 & AGFI & $>0.90$ \\
\hline 7 & CMIN/DF & $<2.00$ \\
\hline 8 & TLI & $>0.95$ \\
\hline
\end{tabular}

Source: Ferdinand (2000). 


\section{RESEARCH RESULT}

Respondent Profile

Respondents in this study were 200 teachers of SDN $3 \mathrm{~T}$ region, described as follows.

Table 1. Respondent Profile

\begin{tabular}{|c|c|c|c|c|}
\hline No & \multicolumn{2}{|c|}{ Description } & $\square$ & $\%$ \\
\hline \multirow{2}{*}{1} & \multirow{2}{*}{ Gender } & Man & 107 & $53.5 \%$ \\
\hline & & Woman & 91 & $46.5 \%$ \\
\hline \multicolumn{3}{|c|}{ Amount } & 200 & $100 \%$ \\
\hline \multirow{4}{*}{2} & \multirow{4}{*}{ Age } & $17-27 \mathrm{Yr}$ & 13 & $6.5 \%$ \\
\hline & & $>27-37$ Yrs & 72 & $36 \%$ \\
\hline & & $>37-47 \mathrm{Yr}$ & 67 & $33.5 \%$ \\
\hline & & $>47 \mathrm{Yrs}$ & 48 & $24 \%$ \\
\hline \multicolumn{3}{|c|}{ Amount } & 200 & $100 \%$ \\
\hline \multirow{2}{*}{3} & \multirow{2}{*}{$\begin{array}{l}\text { Family } \\
\text { Status }\end{array}$} & Marry & 180 & $90 \%$ \\
\hline & & Single & 20 & $10 \%$ \\
\hline \multicolumn{3}{|c|}{ Amount } & 200 & $100 \%$ \\
\hline \multirow{4}{*}{4} & \multirow{4}{*}{ Education } & SMA/SMK/Equivalent & 30 & $15 \%$ \\
\hline & & DII/DIII & 49 & $24.5 \%$ \\
\hline & & Bachelor & 118 & $59 \%$ \\
\hline & & Postgraduate & 3 & $1.5 \%$ \\
\hline \multicolumn{3}{|c|}{ Amount } & 200 & $100 \%$ \\
\hline \multirow{4}{*}{5} & \multirow{4}{*}{ Rank/Goal } & PNS Goal II & 36 & $18 \%$ \\
\hline & & PNS Goal III & 87 & $43.5 \%$ \\
\hline & & PNS Goal IV & 7 & $3.5 \%$ \\
\hline & & Honorary & 70 & $35 \%$ \\
\hline \multicolumn{3}{|c|}{ Amount } & 200 & $100 \%$ \\
\hline \multirow{4}{*}{6} & \multirow{4}{*}{$\begin{array}{l}\text { Years of } \\
\text { service }\end{array}$} & $0-5$ Years & 26 & $13 \%$ \\
\hline & & $>5-15$ Years & 107 & $53.5 \%$ \\
\hline & & $>15-25$ Years & 48 & $24 \%$ \\
\hline & & $>25$ Years & 19 & $9.5 \%$ \\
\hline \multicolumn{3}{|c|}{ Amount } & 200 & $100 \%$ \\
\hline \multirow{2}{*}{7} & \multirow{2}{*}{ Salary/Honor } & $<$ Rp.1.000.000 & 68 & $34 \%$ \\
\hline & & $>$ IDR $1,000,000$ - & 3 & $1.5 \%$ \\
\hline
\end{tabular}

\begin{tabular}{|c|c|c|c|c|}
\hline & & IDR $2,000,000$ & & \\
\hline & & $\begin{array}{c}>\text { IDR } 2,000,000- \\
\text { IDR } 3,000,000\end{array}$ & 71 & $35.5 \%$ \\
\hline & & $>$ IDR 3,000,000 & 58 & $29 \%$ \\
\hline \multicolumn{3}{|c|}{ Amount } & 200 & $100 \%$ \\
\hline \multirow{4}{*}{8} & \multirow{4}{*}{$\begin{array}{c}3 \mathrm{~T} \\
\text { allowance }\end{array}$} & $<$ Rp.1.000.000 & 76 & $38 \%$ \\
\hline & & $\begin{array}{c}>\text { IDR } 1,000,000- \\
\text { IDR } 2,000,000\end{array}$ & 17 & $8.5 \%$ \\
\hline & & $\begin{array}{c}>\text { IDR } 2,000,000- \\
\text { IDR } 3,000,000\end{array}$ & 58 & $29 \%$ \\
\hline & & $>$ IDR 3,000,000 & 49 & $24.5 \%$ \\
\hline \multicolumn{3}{|c|}{ Amount } & 200 & $100 \%$ \\
\hline
\end{tabular}

Source: Primary data reprocessed (2021).

\section{Structural Equation Modeling Analysis}

SEM testing is carried out with two kinds of tests: the model suitability test called CFA and the causality significance test through the regression coefficient test. For Confirmatory factor analysis (CFA) or measurement model analysis, the research is carried out in two stages: first, testing the conceptual model, if the results are unsatisfactory, proceed to the second stage by providing modification treatment to the developed model after paying attention to the modification index and justification of the existing theory. Suppose in the second stage unsatisfactory results are still obtained. In that case, the third step is taken by eliminating or dropping variables that do not have a critical ratio (CR) value in the regression weight of 2.00 because this variable is considered not to have the same indicators as other variables. to explain latent variables (Ferdinand, 2014).

According to Hair et al. (2014), the value of construct validity can be observed through the estimated coefficient $(\lambda)$, which is called the loading factor in other terms. This coefficient is valid, which means that the variable observatory used can represent a particular construct when $>0.05$. This study is guided by the provisions recommended by Ferdinand (2014), namely CR $>2.00$ and the estimated coefficient $(\lambda)$ or loading factor recommended by Hair et al. (2014) CR value CR > 2.00 and the higher loading factor will be better. The results of the data processing will be explained as follows:

Table 2. The goodness of Fit Indices(GFI) computational results

\begin{tabular}{|c|c|c|c|c|c|c|}
\hline \multirow{2}{*}{$\begin{array}{l}\text { Variable/ } \\
\text { Dimension }\end{array}$} & \multirow[b]{2}{*}{ Criteria } & \multirow[b]{2}{*}{ Cut Of Value } & \multicolumn{2}{|c|}{ Model Computing Results } & \multicolumn{2}{|c|}{ Model Evaluation } \\
\hline & & & $\begin{array}{c}\text { EARLY } \\
\text { CFA }\end{array}$ & $\begin{array}{c}\text { FINAL } \\
\text { CFA }\end{array}$ & $\begin{array}{c}\text { CFA } \\
\text { BEGINNING }\end{array}$ & $\begin{array}{l}\text { CFA } \\
\text { END }\end{array}$ \\
\hline \multirow{6}{*}{$\begin{array}{c}\text { Organizational } \\
\text { Commitment } \\
(\mathrm{X} 1): \\
\text { Dimensions of } \\
\text { Affective } \\
\text { Commitment } \\
\text { (KA) }(\mathrm{X} 1.1)\end{array}$} & Chi-square & $\begin{array}{c}\text { Expected } \\
\text { small }\end{array}$ & 43,307 & 23,775 & Not good & Well \\
\hline & Probability & 0.05 & 0.002 & 0.205 & Not good & Well \\
\hline & $\mathrm{CMIN/DF}$ & 2.00 & 2.165 & 1.251 & Not good & Well \\
\hline & RMSEA & 0.08 & 0.079 & 0.036 & Well & Very good \\
\hline & GFI & 0.90 & 0.948 & 0.971 & Well & Very good \\
\hline & TLI & 0.95 & 0.915 & 0.982 & Not good & Well \\
\hline
\end{tabular}




\begin{tabular}{|c|c|c|c|c|c|c|}
\hline & AGFI & $\geq 0.90$ & 0.906 & 0.945 & Well & Very good \\
\hline & CFI & 0.95 & 0.939 & 0.988 & Not good & Well \\
\hline \multirow{8}{*}{$\begin{array}{l}\text { Organizational } \\
\text { Commitment } \\
\text { (X1): } \\
\text { Dimensions of } \\
\text { Normative } \\
\text { Commitment } \\
(\mathrm{KN})(\mathrm{X} 1.2)\end{array}$} & Chi-square & $\begin{array}{c}\text { Expected } \\
\text { small }\end{array}$ & 47,646 & 26,604 & Not good & Well \\
\hline & Probability & 0.05 & 0.000 & 0.114 & Not good & Well \\
\hline & CMIN/DF & 2.00 & 2,382 & 1,400 & Not good & Well \\
\hline & RMSEA & 0.08 & 0.086 & 0.046 & Not good & Well \\
\hline & GFI & 0.90 & 0.941 & 0.966 & Well & Very good \\
\hline & TLI & 0.95 & 0.903 & 0.972 & Not good & Well \\
\hline & AGFI & $\geq 0.90$ & 0.893 & 0.935 & Not good & Well \\
\hline & CFI & 0.95 & 0.931 & 0.981 & Not good & Well \\
\hline \multirow{8}{*}{$\begin{array}{l}\text { Organizational } \\
\text { Commitment } \\
\text { (X1): } \\
\text { Dimensions of } \\
\text { Sustainability } \\
\text { Commitment } \\
\text { (X1.3) }\end{array}$} & Chi-square & $\begin{array}{c}\text { Expected } \\
\text { small }\end{array}$ & 44,376 & 23,943 & Not good & Well \\
\hline & Probability & 0.05 & 0.001 & 0.198 & Not good & Well \\
\hline & CMIN/DF & 2.00 & 2,219 & 1,260 & Not good & Well \\
\hline & RMSEA & 0.08 & 0.080 & 0.037 & Not good & Well \\
\hline & GFI & 0.90 & 0.944 & 0.969 & Well & Very good \\
\hline & TLI & 0.95 & 0.903 & 0.980 & Not good & Well \\
\hline & AGFI & $\geq 0.90$ & 0.900 & 0.941 & Not good & Well \\
\hline & CFI & 0.95 & 0.934 & 0.987 & Not good & Well \\
\hline \multirow{8}{*}{$\begin{array}{c}\text { Competency } \\
\text { Variable (X2), } \\
\text { Pedagogic } \\
\text { Competency } \\
\text { Dimension } \\
\text { (X2.1) }\end{array}$} & Chi-square & $\begin{array}{l}\text { Expected } \\
\text { small }\end{array}$ & 53.908 & 32.276 & Not good & Well \\
\hline & Probability & 0.05 & 0.000 & 0.092 & Not good & Well \\
\hline & CMIN/DF & 2.00 & 2,695 & 1,699 & Not good & Well \\
\hline & RMSEA & 0.08 & 0.095 & 0.061 & Not good & Well \\
\hline & GFI & 0.90 & 0.934 & 0.959 & Well & Very good \\
\hline & TLI & 0.95 & 0.884 & 0.952 & Not good & Well \\
\hline & AGFI & $\geq 0.90$ & 0.881 & 0.922 & Not good & Well \\
\hline & CFI & 0.95 & 0.917 & 0.968 & Not good & Well \\
\hline \multirow{8}{*}{$\begin{array}{c}\text { Competency } \\
\text { Variable (X2), } \\
\text { Dimensions of } \\
\text { Personality } \\
\text { Competence(X } \\
\text { 2.2) }\end{array}$} & Chi-square & $\begin{array}{c}\text { Expected } \\
\text { small }\end{array}$ & 33,242 & 18,941 & Not good & Well \\
\hline & Probability & 0.05 & 0.003 & 0.125 & Not good & Well \\
\hline & CMIN/DF & 2.00 & 2,374 & 1.457 & Not good & Well \\
\hline & RMSEA & 0.08 & 0.085 & 0.049 & Not good & Well \\
\hline & GFI & 0.90 & 0.952 & 0.973 & Well & Very good \\
\hline & TLI & 0.95 & 0.884 & 0.962 & Not good & Well \\
\hline & AGFI & $\geq 0.90$ & 0.905 & 0.942 & Well & Very good \\
\hline & CFI & 0.95 & 0.923 & 0.976 & Not good & Well \\
\hline \multirow{8}{*}{$\begin{array}{c}\text { Competency } \\
\text { Variable (X2), } \\
\text { Dimensions of } \\
\text { Social } \\
\text { Competence } \\
\text { (X2.3) }\end{array}$} & Chi-square & $\begin{array}{c}\text { Expected } \\
\text { small }\end{array}$ & 2,777 & Well & & \\
\hline & Probability & 0.05 & 0.249 & - & Well & - \\
\hline & CMIN/DF & 2.00 & 1.388 & - & Well & - \\
\hline & RMSEA & 0.08 & 0.045 & - & Well & - \\
\hline & GFI & 0.90 & 0.993 & - & Well & - \\
\hline & TLI & 0.95 & 0.986 & - & Well & - \\
\hline & AGFI & $\geq 0.90$ & 0.965 & - & Well & - \\
\hline & CFI & 0.95 & 0.995 & - & Well & - \\
\hline
\end{tabular}




\begin{tabular}{|c|c|c|c|c|c|c|}
\hline \multirow{8}{*}{$\begin{array}{c}\text { Competency } \\
\text { Variable (X2), } \\
\text { Dimensions of } \\
\text { Professional } \\
\text { Competence } \\
\text { (X2.4) }\end{array}$} & Chi-square & $\begin{array}{c}\text { Expected } \\
\text { small }\end{array}$ & 20.984 & 13,964 & Not good & Well \\
\hline & Probability & 0.05 & 0.013 & 0.083 & Not good & Well \\
\hline & CMIN/DF & 2.00 & 2,332 & 1,746 & Not good & Well \\
\hline & RMSEA & 0.08 & 0.084 & 0.063 & Not good & Well \\
\hline & GFI & 0.90 & 0.963 & 0.976 & Well & Very good \\
\hline & TLI & 0.95 & 0.924 & 0.957 & Not good & Well \\
\hline & AGFI & $\geq 0.90$ & 0.913 & 0.936 & Well & Very good \\
\hline & CFI & 0.95 & 0.954 & 0.977 & Well & Very good \\
\hline \multirow{8}{*}{$\begin{array}{c}\text { Academic } \\
\text { Atmosphere } \\
\text { Variable (X3) }\end{array}$} & Chi-square & $\begin{array}{c}\text { Expected } \\
\text { small }\end{array}$ & 14,410 & - & Well & - \\
\hline & Probability & 0.05 & 0.108 & - & Well & - \\
\hline & CMIN/DF & 2.00 & 1,801 & - & Well & - \\
\hline & RMSEA & 0.08 & 0.056 & - & Well & - \\
\hline & GFI & 0.90 & 0.974 & - & Well & - \\
\hline & TLI & 0.95 & 0.966 & - & Well & - \\
\hline & AGFI & $\geq 0.90$ & 0.939 & - & Well & - \\
\hline & CFI & 0.95 & 0.980 & - & Well & - \\
\hline \multirow{8}{*}{$\begin{array}{c}\text { Motivation } \\
\text { Variable (Y1), } \\
\text { Physiological } \\
\text { Needs } \\
\text { Dimension } \\
\text { (Y1.1) }\end{array}$} & Chi-square & $\begin{array}{c}\text { Expected } \\
\text { small } \\
\end{array}$ & 0.051 & - & Well & - \\
\hline & Probability & 0.05 & 0.975 & - & Well & - \\
\hline & CMIN/DF & 2.00 & 0.025 & - & Well & - \\
\hline & RMSEA & 0.08 & 0.000 & - & Well & - \\
\hline & GFI & 0.90 & 1,000 & - & Well & - \\
\hline & TLI & 0.95 & 1.039 & - & Well & - \\
\hline & AGFI & $\geq 0.90$ & 0.999 & - & Well & - \\
\hline & CFI & 0.95 & 1,000 & - & Well & - \\
\hline \multirow{8}{*}{$\begin{array}{c}\text { Motivation } \\
\text { Variable (Y1), } \\
\text { Dimension of } \\
\text { Safety or } \\
\text { Security Needs } \\
\text { (Y1.2) }\end{array}$} & Chi-square & $\begin{array}{c}\text { Expected } \\
\text { small } \\
\end{array}$ & 5.103 & 0.087 & Not good & Well \\
\hline & Probability & 0.05 & 0.078 & 0.768 & Well & Very good \\
\hline & CMIN/DF & 2.00 & 2,552 & 0.087 & Not good & Well \\
\hline & RMSEA & 0.08 & 0.091 & 0.000 & Not good & Well \\
\hline & GFI & 0.90 & 0.986 & 1,000 & Well & Very good \\
\hline & TLI & 0.95 & 0.932 & 1.040 & Not good & Well \\
\hline & AGFI & $\geq 0.90$ & 0.931 & 0.998 & Well & Very good \\
\hline & $\mathrm{CFI}$ & 0.95 & 0.977 & 1,000 & Well & Very good \\
\hline \multirow{8}{*}{$\begin{array}{c}\text { Motivation } \\
\text { Variable (Y1), } \\
\text { Affiliation or } \\
\text { Acceptance } \\
\text { Needs } \\
\text { Dimension } \\
\text { (Y1.3) }\end{array}$} & Chi-square & $\begin{array}{c}\begin{array}{c}\text { Expected } \\
\text { small }\end{array} \\
\end{array}$ & 1.154 & - & Well & - \\
\hline & Probability & 0.05 & 0.561 & - & Well & - \\
\hline & CMIN/DF & 2.00 & 0.577 & - & Well & - \\
\hline & RMSEA & 0.08 & 0.000 & - & Well & - \\
\hline & GFI & 0.90 & 0.997 & - & Well & - \\
\hline & TLI & 0.95 & 1.031 & - & Well & - \\
\hline & AGFI & $\geq 0.90$ & 0.999 & - & Well & - \\
\hline & CFI & 0.95 & 0.985 & - & Well & - \\
\hline \multirow{2}{*}{$\begin{array}{c}\text { Motivation } \\
\text { Variable (Y1), } \\
\text { Esteem or }\end{array}$} & Chi-square & $\begin{array}{c}\text { Expected } \\
\text { small }\end{array}$ & 2,713 & - & Well & - \\
\hline & Probability & 0.05 & 0.258 & - & Well & - \\
\hline
\end{tabular}




\begin{tabular}{|c|c|c|c|c|c|c|}
\hline \multirow{6}{*}{$\begin{array}{l}\text { Status Needs } \\
\text { Dimension } \\
\text { (Y1.4) }\end{array}$} & CMIN/DF & 2.00 & 1.365 & - & Well & - \\
\hline & RMSEA & 0.08 & 0.043 & - & Well & - \\
\hline & GFI & 0.90 & 0.993 & - & Well & - \\
\hline & TLI & 0.95 & 0.970 & - & Well & - \\
\hline & AGFI & $\geq 0.90$ & 0.964 & - & Well & - \\
\hline & $\mathrm{CFI}$ & 0.95 & 0.990 & - & Well & - \\
\hline \multirow{8}{*}{$\begin{array}{c}\text { Motivation } \\
\text { Variable (Y1), } \\
\text { Self } \\
\text { Actualization } \\
\text { Dimension } \\
\text { (Y1.5) }\end{array}$} & Chi-square & $\begin{array}{l}\text { Expected } \\
\text { small }\end{array}$ & 1,780 & - & Well & - \\
\hline & Probability & 0.05 & 0.411 & - & Well & - \\
\hline & CMIN/DF & 2.00 & 0.890 & - & Well & - \\
\hline & RMSEA & 0.08 & 0.000 & - & Well & - \\
\hline & GFI & 0.90 & 0.995 & - & Well & - \\
\hline & TLI & 0.95 & 1.006 & - & Well & - \\
\hline & AGFI & $\geq 0.90$ & 0.976 & - & Well & - \\
\hline & CFI & 0.95 & 1,000 & - & Well & - \\
\hline \multirow{8}{*}{$\begin{array}{c}\text { Performance } \\
\text { Variable (Y2), } \\
\text { Planning } \\
\text { Dimension } \\
\text { (Y2.1) }\end{array}$} & Chi-square & $\begin{array}{c}\text { Expected } \\
\text { small }\end{array}$ & 18,024 & 5.172 & Not good & Well \\
\hline & Probability & 0.05 & 0.003 & 0.207 & Not good & Well \\
\hline & CMIN/DF & 2.00 & 3,605 & 1.293 & Not good & Well \\
\hline & RMSEA & 0.08 & 0.117 & 0.039 & Not good & Well \\
\hline & GFI & 0.90 & 0.968 & 0.990 & Well & Very good \\
\hline & TLI & 0.95 & 0.878 & 0.986 & Not good & Well \\
\hline & AGFI & $\geq 0.90$ & 0.903 & 0.962 & Well & Very good \\
\hline & CFI & 0.95 & 0.939 & 0.994 & Not good & Well \\
\hline \multirow{8}{*}{$\begin{array}{l}\text { Performance } \\
\text { Variable (Y2), } \\
\text { Dimensions of } \\
\text { Implementing } \\
\text { Process (Y2.2) }\end{array}$} & Chi-square & $\begin{array}{c}\text { Expected } \\
\text { small }\end{array}$ & 3.889 & - & Well & - \\
\hline & Probability & 0.05 & 0.566 & - & Well & - \\
\hline & CMIN/DF & 2.00 & 0.778 & - & Well & - \\
\hline & RMSEA & 0.08 & 0.000 & - & Well & - \\
\hline & GFI & 0.90 & 0.992 & - & Well & - \\
\hline & TLI & 0.95 & 1.023 & - & Well & - \\
\hline & AGFI & $\geq 0.90$ & 0.976 & - & Well & - \\
\hline & CFI & 0.95 & 1,000 & - & Well & - \\
\hline \multirow{8}{*}{$\begin{array}{c}\text { Performance } \\
\text { Variable (Y2), } \\
\text { Dimension } \\
\text { Assessing } \\
\text { progress (Y2.3) }\end{array}$} & Chi-square & $\begin{array}{c}\text { Expected } \\
\text { small }\end{array}$ & 0.161 & - & Well & - \\
\hline & Probability & 0.05 & 0.922 & - & Well & - \\
\hline & CMIN/DF & 2.00 & 0.081 & - & Well & - \\
\hline & RMSEA & 0.08 & 0.000 & - & Well & - \\
\hline & GFI & 0.90 & 1,000 & - & Well & - \\
\hline & TLI & 0.95 & 1.024 & - & Well & - \\
\hline & AGFI & $\geq 0.90$ & 0.998 & - & Well & - \\
\hline & CFI & 0.95 & 1,000 & - & Well & - \\
\hline
\end{tabular}

Source: primary data reprocessed (2021)

Furthermore, the results of the Loading Factor $(\lambda)$ and Critical Ratio (CR) can be presented in the following table: 
Table 3. Loading Factor ( $\lambda$ ) and Critical Ratio (CR) Measurement of Organizational Commitment Variable (X1) on the Affective Dimension (X1.1)

\begin{tabular}{|c|c|c|c|c|c|}
\hline \multicolumn{2}{|c|}{ Indicator } & $\begin{array}{c}\text { Loading factor } \\
(\lambda)\end{array}$ & $\begin{array}{c}\mathrm{CR} / \\
\mathrm{t}-\mathrm{Calculate}\end{array}$ & Probability $(\mathrm{p})$ & Information \\
\hline $\mathrm{X} 1.1 .1 \leftarrow$ & $\mathrm{X} 1.1$ & 0.707 & 5.107 & 0.000 & Significant \\
\hline $\mathrm{X} 1.1 .2 \leftarrow$ & $\mathrm{X} 1.1$ & 0.792 & 5.265 & 0.000 & Significant \\
\hline $\mathrm{X} 1.1 .3 \leftarrow$ & $\mathrm{X} 1.1$ & 0.699 & 5.089 & 0.000 & Significant \\
\hline $\mathrm{X} 1.1 .4 \leftarrow$ & $\mathrm{X} 1.1$ & 0.641 & 4.938 & 0.000 & Significant \\
\hline $\mathrm{X} 1.1 .5$ & $\mathrm{X} 1.1$ & 0.428 & 4080 & 0.000 & Significant \\
\hline $\mathrm{X} 1.1 .6 \leftarrow$ & $\mathrm{X} 1.1$ & 0.471 & 4.299 & 0.000 & Significant \\
\hline $\mathrm{X} 1.1 .7 \leftarrow$ & $\mathrm{X} 1.1$ & 0.465 & 4.275 & 0.000 & Significant \\
\hline $\mathrm{X} 1.1 .8$ & $\mathrm{X} 1.1$ & 0.411 & & & Significant \\
\hline
\end{tabular}

Source: Data processed, 2021.

Table 4. Table of Loading Factor ( $\lambda$ ) and Critical Ratio (CR) Variable Measurement (X1), Normative Dimension (X1.2)

\begin{tabular}{|c|c|c|c|c|}
\hline Indicator & $\begin{array}{c}\text { Loading factor }( \\
)\end{array}$ & $\begin{array}{c}\text { CR / } \\
\text { t-Calculate }\end{array}$ & $\begin{array}{c}\text { Probability } \\
(\mathrm{p})\end{array}$ & Information \\
\hline $\mathrm{X} 1.2 .1 \longleftarrow \mathrm{X} 1.2$ & 0.466 & 5.429 & 0.000 & Significant \\
\hline $\mathrm{X} 1.2 .2 \longleftarrow \mathrm{X} 1.2$ & 0.468 & 5.446 & 0.000 & Significant \\
\hline $\mathrm{X} 1.2 .3 \longleftarrow \mathrm{X} 1.2$ & 0.459 & 5.374 & 0.000 & Significant \\
\hline $\mathrm{X} 1.2 .4 \longleftarrow \mathrm{X} 1.2$ & 0.446 & 5.235 & 0.000 & Significant \\
\hline $\mathrm{X} 1.2 .5 \longleftarrow \mathrm{X} 1.2$ & 0.687 & 7.394 & 0.000 & Significant \\
\hline $\mathrm{X} 1.2 .6 \longleftarrow \mathrm{X} 1.2$ & 0.766 & 7,940 & 0.000 & Significant \\
\hline $\mathrm{X} 1.2 .7 \longleftarrow 1.2$ & 0.764 & 7,922 & 0.000 & Significant \\
\hline $\mathrm{X} 1.2 .8 \longleftarrow \mathrm{X} 1.2$ & 0.619 & & & Significant \\
\hline
\end{tabular}

Source: Processed Data, 2021.

Table 5: Table of Loading Factor ( ) and Critical Ratio (CR)

Measurement of Organizational Commitment Variable (X1), Dimensions of Sustainable Commitment (X1.3)

\begin{tabular}{|c|c|c|c|c|}
\hline Indicator & Loading factor $(\mathrm{)}$ & CR/t Count & Probability $(\mathrm{p})$ & Information \\
\hline $\mathrm{X} 1.3 .1 \longleftarrow \mathrm{X} 1.3$ & 0.450 & 5,300 & 0.000 & Significant \\
\hline $\mathrm{X} 1.3 .2 \longleftarrow \mathrm{X} 1.3$ & 0.707 & 5.619 & 0.000 & Significant \\
\hline $\mathrm{X} 1.3 .3 \longleftarrow \mathrm{X} 1.3$ & 0.468 & 7.521 & 0.000 & Significant \\
\hline $\mathrm{X} 1.3 .4 \longleftarrow \mathrm{X} 1.3$ & 0.470 & 7.779 & 0.000 & Significant \\
\hline $\mathrm{X} 1.3 .5 \leftarrow \mathrm{X} 1.3$ & 0.720 & 5.497 & 0.000 & Significant \\
\hline $\mathrm{X} 1.3 .6$ & 0.686 & 5.481 & 0.000 & Significant \\
\hline $\mathrm{X} 1.3 .7 \longleftarrow \mathrm{X} 1.3$ & 0.480 & 7.678 & 0.000 & Significant \\
\hline $\mathrm{X} 1.3 .8 \leftarrow 3$ & 0.450 & & & Significant \\
\hline
\end{tabular}

Source: Data processed, 2021.

Table 6: Loading Factor $(\lambda)$ and Critical Ratio (CR)

Measurement of Competency Variables (X2), Dimensions of pedagogic Competence (X2.1)

\begin{tabular}{|c|c|c|c|c|}
\hline Indicator & Loading factor () & CR/t Count & Probability $(\mathrm{p})$ & Information \\
\hline $\mathrm{X} 2.1 .1 \longleftarrow \mathrm{X} 2.1$ & 0.698 & 5.319 & 0.000 & Significant \\
\hline $\mathrm{X} 2.1 .2 \longleftarrow \mathrm{X} 2.1$ & 0.782 & 5.508 & 0.000 & Significant \\
\hline $\mathrm{X} 2.1 .3 \longleftarrow \mathrm{X} 2.1$ & 0.756 & 5.458 & 0.000 & Significant \\
\hline $\mathrm{X} 2.1 .4 \longleftarrow \mathrm{X} 2.1$ & 0.626 & 5.102 & 0.000 & Significant \\
\hline $\mathrm{X} 2.1 .5 \longleftarrow \mathrm{X} 2.1$ & 0.464 & 4.403 & 0.000 & Significant \\
\hline $\mathrm{X} 2.1 .6 \longleftarrow \mathrm{X} 2.1$ & 0.460 & 4.382 & 0.000 & Significant \\
\hline $\mathrm{X} 2.1 .7 \longleftarrow$ X2.1 & 0.447 & 4.314 & 0.000 & Significant \\
\hline $\mathrm{X} 2.1 .8 \longleftarrow$ & & & Significant \\
\hline
\end{tabular}

Source: Data reprocessed, 2021. 
Table 7: Table of Loading Factor $(\lambda)$ and Critical Ratio (CR)

Measurement of Competency Variables (X2), Dimensions of Personality Competence (X2.2)

\begin{tabular}{|c|c|c|c|c|}
\hline Indicator & Loading factor $(\mathrm{)}$ & $\mathrm{CR} / \mathrm{t}$ Count & Probability $(\mathrm{p})$ & Information \\
\hline $\mathrm{X} 2.2 .1 \longleftarrow \mathrm{X} 2.2$ & 0.553 & 5.268 & 0.000 & Significant \\
\hline $\mathrm{X} 2.2 .2 \longleftarrow \mathrm{X} 2.2$ & 0.590 & 5.440 & 0.000 & Significant \\
\hline $\mathrm{X} 2.2 .3 \longleftarrow \mathrm{X} 2.2$ & 0.482 & 4.737 & 0.000 & Significant \\
\hline $\mathrm{X} 2.2 .4 \longleftarrow \mathrm{X} 2.2$ & 0.535 & 5.157 & 0.000 & Significant \\
\hline $\mathrm{X} 2.2 .5 \longleftarrow \mathrm{X} 2.2$ & 0.581 & 5.424 & 0.000 & Significant \\
\hline $\mathrm{X} 2.2 .6 \longleftarrow$ X2.2 & 0.598 & 5.512 & 0.000 & Significant \\
\hline $\mathrm{X} 2.2 .7 \longleftarrow$ & & & Significant \\
\hline
\end{tabular}

Source: Data processed, 2021

Table 8: Table of Loading Factor $(\lambda)$ and Critical Ratio (CR)

Measurement of Competency Variables (X2), Dimensions of Social Competence (X2.3)

\begin{tabular}{|c|c|c|c|c|}
\hline Indicator & Loading factor () & CR/t Count & Probability $(\mathrm{p})$ & Information \\
\hline $\mathrm{X} 2.3 .1$ & 0.712 & 5.735 & 0.000 & Significant \\
\hline $\mathrm{X} 2.3 .2 .3$ & 0.661 & 5.579 & 0.000 & Significant \\
\hline $\mathrm{X} 2.3 .3-32.3$ & 0.751 & 4,801 & 0.000 & Significant \\
\hline $\mathrm{X} 2.3 .4-\mathrm{X} 2.3$ & 0.499 & & & Significant \\
\hline
\end{tabular}

Source: Primary Data Reprocessed, 2021.

Table 9: Table of Loading Factor $(\lambda)$ and Critical Ratio (CR)

Measurement of Competency Variables (X2), Dimensions of Professional Competence (X2.4)

\begin{tabular}{|c|c|c|c|c|}
\hline Indicator & Loading factor ( ) & CR/t Count & Probability (p) & Information \\
\hline $\mathrm{X} 2.4 .1$ & 0.655 & 6.353 & 0.000 & Significant \\
\hline $\mathrm{X} 2.4 .2$ & 0.664 & 6.657 & 0.000 & Significant \\
\hline $\mathrm{X} 2.4 .3$ & S2.4 & 6.651 & 0.000 & Significant \\
\hline $\mathrm{X} 2.4 .4$ \\
\hline $\mathrm{X} 2.4 .52 .4$ & 0.583 & 6.109 & 0.000 & Significant \\
\hline $\mathrm{X} 2.4 .6$ \\
\hline
\end{tabular}

Source: Data processed, 2021.

Table 10: Loading Factor $(\lambda)$ and Critical Ratio (CR)

Measurement of Academic Atmosphere Variables (X3)

\begin{tabular}{|c|c|c|c|c|}
\hline Indicator & Loading factor $(\lambda)$ & CR/t Count & Probability $(\mathrm{p})$ & Information \\
\hline X3.1 & 0.687 & 7.062 & 0.000 & Significant \\
\hline X3.2 & 0.684 & 7.042 & 0.000 & Significant \\
\hline X3.3 & 0.658 & 6.868 & 0.000 & Significant \\
\hline X3.4 & 0.610 & 6.516 & 0.000 & Significant \\
\hline $\mathrm{X} 3.5$ & 0.471 & 5.317 & 0.000 & Significant \\
\hline $\mathrm{X} 3.6 \longleftarrow$ X3 & 0.620 & & & Significant \\
\hline
\end{tabular}

Source: Data processed, 2021. 
Table 11: Loading Factor $(\lambda)$ and Critical Ratio (CR)

Measurement of Motivation Variables (Y1), Dimensions of Physiological Needs (Y1.1)

\begin{tabular}{|c|c|c|c|c|}
\hline Indicator & Loading factor ( ) & CR/t Count & Probability (p) & Information \\
\hline Y1.1.1 Y1.1 & 0.606 & 6.372 & 0.000 & Significant \\
\hline Y1.1.2 Y1.1 & 0.668 & 6.209 & 0.000 & Significant \\
\hline Y1.1.3 Y1.1 & 0.728 & 5.892 & 0.000 & Significant \\
\hline Y1.1.4 Y1.1 & 0.591 & & & Significant \\
\hline
\end{tabular}

Source: Data processed, 2021.

Table 12: Loading Factor $(\lambda)$ and Critical Ratio (CR)

Measurement of Motivation Variables (Y1), Dimensions of Safety or Security Needs (Y1.2)

\begin{tabular}{|c|c|c|c|c|}
\hline Indicator & Loading factor ( ) & CR/t Count & Probability (p) & Information \\
\hline Y1.2.1 & 0.476 & 5.554 & 0.000 & Significant \\
\hline Y1.2.2 & 0.853 & 4.911 & 0.000 & Significant \\
\hline Y1.2.3 & 0.548 & 5.404 & 0.000 & Significant \\
\hline Y1.2.4 Y1.2 & 0.542 & & & Significant \\
\hline
\end{tabular}

Source: Data processed, 2021.

Table 13: Loading Factor $(\lambda)$ and Critical Ratio (CR)

Measurement of Motivation Variables (Y1), Affiliation or Acceptance Needs Dimensions (Y1.3)

\begin{tabular}{|c|c|c|c|c|}
\hline Indicator & Loading factor ( ) & CR/t Count & Probability (p) & Information \\
\hline Y1.3.1 Y1.3 & 0.696 & 4.649 & 0.000 & Significant \\
\hline Y1.3.2 Y1.3 & 0.490 & 4.498 & 0.000 & Significant \\
\hline Y1.3.3 Y1.3 & 0.449 & 4.165 & 0.000 & Significant \\
\hline Y1.3.4 1.3 & 0.561 & & & Significant \\
\hline
\end{tabular}

Source: Data processed, 2021.

Table 14: Loading Factor $(\lambda)$ and Critical Ratio (CR)

Measurement of Motivation Variables (Y1), Esteem Dimensions or Status Needs (Y1.4)

\begin{tabular}{|c|c|c|c|c|}
\hline Indicator & Loading factor ( ) & CR/t Count & Probability (p) & Information \\
\hline Y1.4.1 Y1.4 & 0.500 & 4069 & 0.000 & Significant \\
\hline Y1.4.2 Y1.4 & 0.519 & 4.126 & 0.000 & Significant \\
\hline Y1.4.3 Y1.4 & 0.582 & 4.253 & 0.000 & Significant \\
\hline Y1.4.4 & 0.546 & & & Significant \\
\hline
\end{tabular}

Source: Data processed, 2021.

Table 15: Loading Factor $(\lambda)$ and Critical Ratio (CR)

Measurement of Motivation Variable (Y1), Self Actualization Dimension (Y1.5)

\begin{tabular}{|c|c|c|c|c|}
\hline Indicator & Loading factor ( ) & $\begin{array}{c}\text { CR / } \\
\text { t-Calculate }\end{array}$ & Probability (p) & Information \\
\hline Y1.5.1 Y1.5 & 0.621 & 5.117 & 0.000 & Significant \\
\hline Y1.5.2 Y1.5 & 0.580 & 5,000 & 0.000 & Significant \\
\hline Y1.5.3 Y1.5 & 0.693 & 5.257 & 0.000 & Significant \\
\hline Y1.5.4 Y1.5 & 0.524 & & & Significant \\
\hline
\end{tabular}

Source: Data processed, 2021. 
Table 16: Loading Factor $(\lambda)$ and Critical Ratio (CR)

Measurement of Performance Variables (Y2), Planning Dimensions (Y2.1)

\begin{tabular}{|c|c|c|c|c|}
\hline Indicator & $\begin{array}{c}\text { Loading factor ( } \\
\text { ( }\end{array}$ & $\begin{array}{c}\text { CR / } \\
\text { t-Calculate }\end{array}$ & Probability (p) & Information \\
\hline Y2.1.1 Y2.1 & 0.597 & 5.330 & 0.000 & Significant \\
\hline Y2.1.2 Y2.1 & 0.694 & 5.147 & 0.000 & Significant \\
\hline Y2.1.3 Y2.1 & 0.655 & 5.558 & 0.000 & Significant \\
\hline Y2.1.4 Y2.1 & 0.718 & 5.738 & 0.000 & Significant \\
\hline Y2.1.5 Y2.1 & 0.516 & & & Significant \\
\hline
\end{tabular}

Source: Data processed, 2021.

Table 17: Loading Factor $(\lambda)$ and Critical Ratio (CR)

Measurement of Performance Variables (Y2), Dimensions of Implementing Processes (Y2.2)

\begin{tabular}{|c|c|c|c|c|}
\hline Indicator & $\begin{array}{c}\text { Loading factor } \\
(\lambda)\end{array}$ & CR/t Count & Probability $(\mathrm{p})$ & Information \\
\hline $\mathrm{Y} 2.2 .1 \longleftarrow \mathrm{Y} 2.2$ & 0.522 & 4,571 & 0.000 & Significant \\
\hline $\mathrm{Y} 2.2 .2 \longleftarrow \mathrm{Y} 2.2$ & 0.496 & 4.444 & 0.000 & Significant \\
\hline $\mathrm{Y} 2.2 .3 \longleftarrow \mathrm{Y} 2.2$ & 0.406 & 3.901 & 0.000 & Significant \\
\hline $\mathrm{Y} 2.2 .4 \longleftarrow \mathrm{Y} 2.2$ & 0.618 & 4.875 & 0.000 & Significant \\
\hline $\mathrm{Y} 2.2 .5 \longleftarrow \mathrm{Y} 2.2$ & 0.563 & & & Significant \\
\hline
\end{tabular}

Source: Data processed, 2021.

Table 18: Loading Factor ( ) and Critical Ratio (CR)

Measurement of Performance Variables (Y2), Dimensions of Assessing Progress (Y2.3)

\begin{tabular}{|c|c|c|c|c|}
\hline Indicator & Loading factor () & CR/t Count & Probability $(\mathrm{p})$ & Information \\
\hline Y2.3.1 & 0.685 & 7.251 & 0.000 & Significant \\
\hline Y2.3.2 & 0.834 & 7,934 & 0.000 & Significant \\
\hline Y2.3.3 Y2.3 & 0.726 & 7,531 & 0.000 & Significant \\
\hline Y2.3.4 Y2.3 & 0.614 & & & Significant \\
\hline
\end{tabular}

Source: Data processed, 2021.

Based on Tables 3 to 18 above, it shows that all indicators of the variable/dimension loading factor meet the requirements (LF 0.40). This illustrates that all of these indicators have contributed to the variable dimensions. Next for the probability value of each value is 0.000 . it means that it has fulfilled the requirements ( $\mathrm{p}$ 0.05). This indicates that the indicator statements used in this study on the variable dimensions are significantly indicators of the formed factors.

\section{Hypothesis test}

To test the hypothesis of the Effect of Organizational Commitment, Competence, Academic Atmosphere on Motivation and Performance in Tojo Una-Una Regency (Study on State Elementary School Teachers in Region 3 T: Disadvantaged, Foremost, Outermost), can be presented presented in path coefficients showing the influence between these variables (Table 19)

Table 19: Hypothesis Testing The Effect of Organizational Commitment, Competence, Academic Atmosphere on Motivation and Performance in Tojo Una-Una Regency (Study on State Elementary School Teachers in Region 3 T: Disadvantaged, Foremost, Outermost).

\begin{tabular}{|l|l|l|l|l|}
\hline Track & $\begin{array}{l}\text { Regression } \\
\text { Weight }\end{array}$ & $\begin{array}{l}\text { Critical } \\
\text { Ratio }\end{array}$ & $\begin{array}{l}\text { Probability } \\
(\mathrm{p})\end{array}$ & Information \\
\hline Organizational Commitment $(\mathrm{X} 1) \rightarrow$ Motivation $(\mathrm{Y} 1)$ & 0.815 & 4.489 & $* * *$ & Significant \\
\hline Competence $(\mathrm{X} 2) \rightarrow$ Motivation $(\mathrm{Y} 1)$ & 0.003 & 0.275 & .784 & Not significant \\
\hline Academic Atmosphere $(\mathrm{X} 3) \rightarrow$ Motivation $(\mathrm{Y} 1)$ & 0.058 & 2.266 & .023 & Significant \\
\hline Organizational Commitment $(\mathrm{X} 1) \rightarrow$ Performance $(\mathrm{Y} 2)$ & 0.481 & 6,861 & $* * *$ & Significant \\
\hline Competence $(\mathrm{X} 2) \rightarrow$ Performance $(\mathrm{Y} 2)$ & -0.013 & -1.412 & .158 & Not significant \\
\hline Academic Atmosphere $(\mathrm{X} 3) \rightarrow$ Performance $(\mathrm{Y} 2)$ & 0.066 & 2,594 & .009 & Significant \\
\hline Motivation $(\mathrm{Y} 1) \rightarrow$ Performance $(\mathrm{Y} 2)$ & 0.138 & 2,619 & .009 & Significant \\
\hline
\end{tabular}

Source: Processed SEM Data, AMOS, 20.0. 


\section{DISCUSSION}

\section{The effect of organizational commitment on motivation}

The hypothesis test results (H1) stated that there was a significant influence between organizational commitment and motivation. This study supports the findings of Farida, et al, (2016), Bano et al (2019) and Tugrul (2013), Yanti \& Sanny.,(2019) and Diansyah et al., (2020). The results of this study are different from the findings of research conducted by Hari et al. (2021), which explains that there is no significant effect between organizational commitment and teacher work motivation. Study Hari et al., (2021) In measuring commitment, it is not based on the division by Dimension, namely the dimensions of affective dedication, normative commitment, and ongoing commitment.

Furthermore, the findings in this research explain that organizational commitment consists of affective, normative, and continual commitment dimensions. It was revealed that teachers in the $3 \mathrm{~T}$ region, SDN teachers have a high affective obligation, especially teachers who have worked in the $3 \mathrm{~T}$ region between 5-15 years (54\%) and above 15-25 years (24\%). Zacher \& Rudolph (2021) and Karimah et al. (2021) explain the psychological contract on emotional involvement and career-related behavior through two dimensions of the future time perspective of work. Associated with economic value or compensation benefits received by the opinion of Robbins \& Timothy (2015), explains loyalty related to material compensation guarantees other than the level of trust.

\section{The influence of Competence on motivation}

The hypothesis test results $(\mathrm{H} 2)$ stated that there was no significant effect between Competence and motivation. This research is not in line with the study conducted by Heriyanto et al. (2018), Utoyo et al. (2019), Tarigan \& Setiawan (2020), and Katarina Hutagalung et al. (2020)found that employee competence had a significant effect on increasing employee motivation. However, the findings on the object of this study were not proven. According to Boyatzis (1982), in theory, Competence refers to the knowledge-based performance that integrates practical and theoretical knowledge with personal and social qualities.

Furthermore, what is sufficient to support the implementation of teaching process activities is the professional Competence of teachers who are categorized as enough. However, their existence is very limited to the support of learning facilities and infrastructure and access to destinations. This finding is in line with Fahmi et al. (2021), who explained that professional Competence has a positive and significant effect on teacher performance. This study also found that work motivation is the most dominant variable influencing teacher performance improvement in schools.

\section{The influence of the academic atmosphere on motivation}

The hypothesis test results $(\mathrm{H} 3)$ stated that there was a significant influence between the academic atmosphere on work motivation. This study is in line with the research findings conducted by Ahmad et al. (2018) andAshraf, (2019)confirmed that the work atmosphere has a significant mediating role in linking supervisor cooperation and employee job satisfaction in the organization. In line with the same opinionAgung Nugroho et al. (2020) and Sutanto et al. ( 2018)that organizational justice and work environment have a positive and significant effect on employee motivation, both individually and collectively. The findings of this study can be interpreted that the better the academic Susana, the higher the work motivation of teachers who work in the $3 \mathrm{~T}$ area.

\section{The effect of organizational commitment on performance}

The hypothesis test results (H4) prove that organizational commitment has a significant effect on performance. This study supports the results of previous research conducted byRamli, (2019), which states that high organizational commitment turns out to have a positive and significant effect on employees. Likewise, research Oyewobi et al. (2019), Rivai et al., (2019), Loan (2020). Rahmanullah et al., (2021) and Yekti Irawan \& Usman, (2021). The results of this study can be interpreted that the higher organizational commitment will impact the high performance of teachers following government expectations, especially for teachers who work in areas that are completely limited in terms of facilities and infrastructure.

Performance improvement is seen in the organizational commitment that puts forward affective, normative, and ongoing commitments to improve performance, especially in work planning, implementation processes, and assessing progress. More objective evidence will emerge from examining the materials used and prepared by the instructor and the performance that students have shown in their final gradeslvarez-Gil, (2021). Theoretically, according to Robbins \& Timothy (2015), employee performance is a function of the interaction between ability and motivation. Every employee in the organization is required to make a positive contribution through good performance, considering that organizational performance depends on the performance of its employees. (Gibson et al.,; 2010 and Simamora, 2012).

\section{The influence of Competence on performance}

The hypothesis test results (H5) stated that it was not proven that Competence had a significant effect on performance. The results of this study are different from the findings of Ayu et al. (2018), Sammy et al. (2019), and Tarigan \& Setiawan (2020)stated that Competence has an impact on improving organizational performance. In addition, it was found that employee competence and organizational commitment dimensions showed a positive and significant influence on employee performance. This is in line with the findingsParashakti et al. (2020). The results of the path analysis show that the Work Environment and Competence have a positive and significant effect on performance, either directly or indirectly through motivation. 
Furthermore, theoretically, Competence proposed by Boyatzis (1982), Brockman et al. (1993) refers to the knowledge-based performance that integrates practical and theoretical knowledge with personal and social qualities. This can be shown in the findings of this research that the competencies related to planning performance achieved in the $3 \mathrm{~T}$ work area can be explained that in carrying out the work assisted by learning tools, formulating objectives and indicators of learning success, preparing scenarios for learning activities, carrying out plans and procedures, preparing assessment tools, and planning guidance and counseling programs.

\section{The Influence of Academic Atmosphere on Performance}

The hypothesis test results (H6) state that it is proven that the academic atmosphere has a significant effect on performance. This study supports the results of research conducted bySuryawan et al. (2020), which states that the intellectual atmosphere substantially affects performance and the environment. Likewise, a study conducted by Pharisees \& Fani (2019), Fachmi et al. (2021)states a positive and significant influence between work environment variables on employee performance. In theory, according to Danang (2015), the work environment is everything that is around the workers, and that can affect him in carrying out the assigned tasks.

Regarding the work environment that can affect the performance of teachers on duty in the $3 \mathrm{~T}$ area, despite the limitations of facilities and infrastructure and access to transportation, the teachers continue to carry out their duties faithfully. It is proven that almost 80 percent of the employees remain in the area, which shows and maintains the value of responsibility for work, upholding the importance of honesty in work, respecting the matter of criticality in work, maintaining the value of perseverance in carrying out work, having a high curiosity value towards educational progress, and having high importance of caring for students.

\section{The influence of motivation affects performance.}

The hypothesis test results (H7) stated that it was proven that the academic motivation variable had a significant effect on performance. This study supports the results of research in line with research conducted by Zameer et al. (2014), Gachengo \& Wekesa (2017), Kadek et al. (2021), Nasution et al. (2021), and Rijanti et al. (2021) states that motivation has a positive effect on organizational performance. In addition, find, motivation plays a vital role in all public and private organizations, and without motivation, employees cannot achieve organizational goals. The support for motivation theory put forward by Robbins and Judge (2015) explains that motivation is a process of strength and perseverance to achieve the desired goal.

In addition, the motivation for the dimensions of Safety or Security Needs is fulfilled. This means that respondents feel safe while on duty in the $3 \mathrm{~T}$ area, such as the availability of access to transportation from their place of residence to the district capital. In addition, there is a guarantee of security against the certainty of guaranteeing the consistency of the rules for remote areas allowances. Likewise, it was identified that motivation with the dimensions of social needs (Affiliation or Acceptance Needs) was well established. They felt that social relations were well established between leaders, co-workers, and the surrounding community.

\section{CONCLUSION}

Based on the results and discussion of the research, it can be concluded as follows: (1) organizational commitment has a significant effect on teacher motivation in SDN Region $3 \mathrm{~T}$ (Lagging, Foremost, Outermost), (2) competence has no significant effect on motivation, (3) academic atmosphere has a significant effect on motivation, (4) organizational commitment has a significant effect on performance, (5) competence has no significant effect on performance, (6) academic atmosphere has a significant effect on teacher performance, and (7) motivation has a significant effect on performance.

The suggestions in this research are: (1) increasing organizational commitment, especially on sustainable commitments related to guaranteeing economic benefits while serving in the $3 \mathrm{~T}$ (Lagged, Frontier, Outermost) regions, it is necessary to review the number of remote area allowances (Dacil) and facilitate monthly payments, (2) increase pedagogic Competence, especially in increasing the ability to excel and mastering information technology, especially anticipating online/offline learning, (3) maintaining the academic atmosphere, especially in increasing responsibility for tasks and functions and showing perseverance in work, and (4) ensuring psychological needs ( clothing, food), related to the smooth process of disbursement of salaries/honors and allowances for Dacil (remote areas).

\section{REFERENCES}

[1] Agustina, Zubair, Desiana, Vidayanti, Endi Rekarti, dan Sri, Hesti. (2016). Development Guidelines For Academic Atmosphere. Jakarta: Mercu Buana.

[2] Ahmad, Suriansyah dan Aslamiah (2018). Teacher's Job Satisfaction On Elementary School: Relation To Learning Environment. The Open Psychology Journal, 2018, 11, 123-130.

[3] Alafy, Hany R., Ibrahim S. Al-Aodah, dan Emad A. Shalaby (2013). Leadership Skills in Higher Education. Mediterranean Journal of Social Sciences, Vol. 4, No. 2, hal. 425- 480.

[4] Allen, S., and Meyer, G., (2009). Organization Commitment in Management Perspective. Published by Prentice Hall, New York.

[5] Álvarez-Gil, F. J. (2021). Essential Framework For Planning Clil Lessons And Teachers' Attitudes Toward The Methodology. In Teaching Language And Content In Multicultural And Multilingual Classrooms. (Pp. 315-338). Springer International Publishing.

[6] Anwar, Khoirul., Muhammad, Havidz, Aima (2019). Dinasti International Journal of Education Management and Social Science, Vol 1 No 2 (2019).

[7] Ashraf, M. A. (2019). The Mediating Role Of Work Atmosphere In The Relationship Between Supervisor Cooperation, Career Growth And Job Satisfaction. Journal Of Workplace Learning, 31(2), 78-94. 
[8] Atiya, Inayatullah dan Palwasha, Jehangir. (2016). Teacher's Job Performance: The Role of Motivation. Journal of Social Sciences Vol. 5 No. 2.

[9] Ayu, I., Martini, O., Ketut Rahyuda, I., Sintaasih, D. K., \& Piartrini, P. S. (2018). The Influence Of Competency On Employee Performance Through Organizational Commitment Dimension Want More Papers Like This? The Influence Of Competency On Employee Performance Through Organizational Commitment Dimension. 20, 29-37.

[10] Bachrudin, A., \& Harapan L. T. (2003). Analisis Data Untuk Penelitian Survai dengan Menggunakan Lisrel 8. Bandung: Jurusan Statistika FMIPA - Unpad.

[11] Bambang, Sulistyo, Maria Magdalena Minarsih, dan M Mukeri Warso. (2016). Pengaruh Pendidikan Dan Latihan Profesi Guru. Kedisiplinan Guru, Dan Kompetensi Guru Terhadap Kinerja Guru Di Smp Masehi Jepara. Journal Of Management, Volume 2 No.2 Maret 2016

[12] Bano, Khushnuma; Azra Ishrat, KK Mishra. (2019). Factors Affecting Organizational Commitment Of Teachers In Government And Private Universities. International Journal of Scientific \& Technology Research Volume 8, Issue 11, November 2019 Issn 2277-8616.

[13] Basilius Redan Werang dan Anak Agung Gede Agung (2017). Teachers' job satisfaction, organizational commitment, and performance in Indonesia: A Study from Merauke District, Papua. International Journal of Development and Sustainability ISSN: 2186-8662 - www.isdsnet.com/ijds Volume 6 Number 8 (2017): Pages 700-711 ISDS Article ID: IJDS17080706.

[14] Bosque dan Dore .(1998). Environment and Apparatuship Information. Journal of Distance Education, 13(1) ISSN 08300445 .

[15] Boyatzis, R.E.. (1982). The Competent Manager: A Model of Effective New York: John Wiley \& Sons.

[16] Brockman, R.P. (1993). Glucose and Short Chain Fatty Acid Metabolism. In: J.M Forbes \& J. France (Eds.).Quantitative Aspects of Ruminant Digestion

Metabolism. CAB. International, Wallingford

[17] Danang Sunyoto. (2015). Manajemen dan Pengembangan Sumber Daya Manusia. Yogyakarta: Center for Academic Publishing Service.

[18] Diansyah, M., Sudiartha Athar, H., \& Fauzi, A. (2020). Kompetensi Dan Komitmen Organisasi Kaitanya Pada Motivasi Dan Kinerja Pegawai. (Vol. 8, Issue 2).

[19] Elizabeth N. Nyakundi (2019). Influence of Teacher Motivation to Academic Performance of Pupils in Primary Schools in Kenya International Journal of Applied Psychology 2019, 9(2): 74-79.

[20] Fachmi, M., Mustafa, M., \& Ngandoh, A. M. (2021). The Role Of Motivation And Professional Competence In Improving Teacher Performance. Journal Of Digital Learning And Education, 1(01), $39-46$.

[21] Farida, Syarifah Ida, Muhammad, Iqbal, Augustina, Kurniasih. (2016). Pengaruh Kepercayaan Dan Komitmen Organisasi Terhadap Motivasi Dan Kepuasan Kerja. Jurnal Kependidikan, Volume 46, Nomor 1, Mei 2016, Halaman 121-134.

[22] Farisi, S., \& Fani, W. M. (2019). Influence Of Work Environment And Work Discipline On Employee Performance. International Conference On Global Education, 69-81.

[23] Fei, Evonne Lai Eng; Crispina, Gregory K Han. (2019). The Influence Of School Climate, Teachers' Commitment, Teachers' Motivation On Eachers' Work Performance Through Teachers' Job Satisfaction.

[24] Ferdinand, A. (2000). Structural Equation Modelling dalam Penelitian Manajemen. Semarang : Penerbit Universitas Diponegoro.

[25] Ferdinand, Augusty. 2014. Metode Penelitian Manajemen. Edisi 5. Semarang : 60 Badan Penerbit Universitas Diponegoro.

[26] Gachengo, V., \& Wekesa, S. (2017). Influence Of Motivation On Employee Performance: A Case Of National Bank Of Kenya. Int. J. Bus. Manag. Soc. Res, 03(02), 179-185.
[27] Gibson, James L., John M. Ivancevich \& James H. Donnelly, Jr. (2010). Organisasi, Perilaku, Struktur, Proses. (Alih Bahasa Nunuk Adiarni), Penerbit Binarupa Aksara, Jakarta.

[28] Gibson, JamesL, John M. Ivancevich, James H. Jr. Donnelly, and Robert Konopaske. (2011). Organizations: Behavior, Structure, Processes. Fourteenth edition. McGraw-Hill. New York

[29] Hafeez, Igra., Zhu, Yingjun. , Saba, Hafeez., Rafiq Mansoor., Khaliq Ur Rehman. (2019). Impact Of Workplace Environment On Employee Performance: Mediating Role Of Employee Health. Business, Management and Education ISSN 2029-7491 / eISSN 2029-6169 2019 Volume 17 Issue 2: 173-193.

[30] Hair et.al. (2014). A Primer On Partial Least Squares Structural Equation Modeling (Pls Sem). USA: SAGE.

[31] Hamidi, Busri Endang, M. Chiar. (2017). Motivasi Guru Bertahan Mengajar Di Daerah Terpencil. Program Magister Administrasi Pendidikan FKIP Untan Pontianak.

[32] Haniatin, Nabila, (2016). The Influence Of Pedagogic Competence And Professional Competence To Performance Of Teachers Social Studies In Trowulan District. International Conference on Ethics of Business, Economics, and Social Science. ICEBESS 2016 Proceeding. ISSN: $2528-617 \mathrm{X}$

[33] Hari, L., Mardi, N., Hartati, C. S., Wijaya, U., \& Surabaya, P. (2021). Pengaruh Komitmen, Komunikasi Dan Kompetensi Terhadap Kinerja Guru Melalui Motivasi Kerja Guru Smk Negeri Dander Kabupaten Bojonegoro. Jmp Online, 5(2), 126-141.

[34] Hasibuan. M. (2013). Manajemen Sumber Daya Manusia. Jakarta: Bumi Aksara.

[35] Heriyanto, Tri.,Jamal, Abdul, Naser., Kohar, Adi Setia. (2018). The Effect Of Competence And Discipline On Work Motivation And Impact On Employee Performance Of Pratama Tax Office In Malang Utara.

[36] Jafri, M. H. and Lhamo, T. (2013) 'Organizational Commitment and Work Performance in Regular and Contract Faculties of Royal University of Bhutan. Journal of Contemporary Research in Management, Vol. 8(2), pp. 47-58.

[37] Kadek, N., Pahlawan, I. I., \& Rasmini, N. K. (N.D.). The Effect Of Situational Leadership Style And Motivation On Organizational Performance With Internal Locus Of Control As Moderation Variables (Studies At Lembaga Perkreditan Desa In Abiansemal District) In American. Journal Of Humanities And Social Sciences Research (Issue 5).

[38] Karimah, R. P., Abdullah, S., \& Setiadi, R. (2021). Analysis Of Factors Affecting Employee Loyalty Of Pt X In Jakarta Region. Journal Of Physics: Conference Series, 1725(1), 12091

[39] Katarina Hutagalung, R., Nadear Purba, E., Tungkir Silalahi, J., Putri, A. (2020). The Effect Of Competence, Work Ethic, Work Discipline, And Work Motivation On Performance Of Government Employee In Mentawai Islands District Health Office. International Journal Of Innovative Science And Research Technology (Vol. 5, Issue 8).

[40] Keputusan Menteri Pendidikan dan Kebudayaan Republik Indonesia Nomor 123/P/2012 Tentang Penetapan Daerah Khusus.

[41] Kreitner Robert \& Angelo Kinicki (2014). Perilaku Organisasi. Jakarta: Salemba Empat.

[42] Kurniawan. (2013). Model Pengembangan Atmosfer Akademik: Pembentukan Iklim Kampus Yang Beretika Dan Bermoral Conference: Seminar Nasional \& Call For Paper FMI ke-5, At Pontianak, Kalimantan Barat, Indonesia.

[43] Kusnendi. (2005). Analisis Jalur Konsep dan Aplikasi dengan Program SPSS dan Lisrel 8. Bandung: UPI.

[44] Loan, L. T. M. (2020). The Influence Of Organizational Commitment On Employees' Job Performance: The Mediating Role Of Job Satisfaction. Management Science Letters, 10(14), $3307-3312$

[45] Maende, J.B dan Luvai, O.N. (2014). Influence of Teacher Professional Development on the Use of Educational Resources in Public Secondary Schools in Kenya. International Journal of Education and Research Vol. 2 No. 4 April 2014. 
[46] McClelland, David (1973). McClelland, D. (1973). Testing for competence rather than for intelligence. American Psychologist, Vol.28, No1, pp.1-14.

[47] Nasution, M., Yeni, S., Yondra, A., \& Putri, A. (N.D.). The Influence Of Organizational Structure And Job Analysis On Work Motivation And Its Impact On The Performance Of The Office Of Cooperatives For Small And Medium Enterprises, Industry And Trade (Koperindag Mentawai Islands Regency in American. Journal Of Humanities And Social Sciences Research (Issue 5).

[48] Nawangsih, Sri Kandariyah dan Fitria Linayaningsih. (2015). Hubungan antara Motivasi dan Kinerja Guru di SMA "X". $J$. DINAMIKA SOSBUD Volume 17 Nomor 2, Juni $2015: 117-121$.

[49] Oyewobi, L. O., Oke, A. E., Adeneye, T. D., \& Jimoh, R. A. (2019). Influence Of Organizational Commitment On Work-Life Balance And Organizational Performance Of Female Construction Professionals. Engineering, Construction And Architectural Management, 26(10), 2243-2263.

[50] Parashakti, R. D., Fahlevi, M., Ekhsan, M., \& Hadinata, A. (2020). The Influence Of Work Environment And Competence On Motivation And Its Impact On Employee Performance In Health Sector. 259-267.

[51] Rahmanullah, F., Barliana, M. S., Meirawan, D., \& Maknun, J. (2021). An Evaluation Of Educational Facilities And Infrastructure In Vocational High School. 248-252.

[52] Ramli, A. H. (2019). Organizational Commitment And Employee Performance At Distributor Companies. Business And Entrepreneurial Review, 17(2), 121.

[53] Raziq, \& Raheela Maulabakhsh. (2015). Impact of Working Environment on Job Satisfaction. Procedia Economics and Finance, 717-725.

[54] Rhini Fatmasari et al (2017). The Effect Of Teacher's Competency, Achievement Motivation And Creativit Their Teaching Performance. International Journal of Current Research Vol. 9, Issue, 12, pp.63541-63544.

[55] Rijanti, T., Kasmari, Prabowo, R. E., \& Sukmaningrum, D. (2021). The Role Of Moderation Of Organizational Culture On The Effect Of Motivation And Compensation On Employee Performance. Proceedings Of The 3rd. International Conference On Banking, Accounting. Management And Economics (Icobame 2020), 169, 368-372.

[56] Rivai, H. A., Reza, D. Y., \& Lukito, H. (2019). Distributive Justice, Job Satisfaction And Organizational Commitment As Antecedents Of Employee Performance: A Study In Indonesia National Health Insurance Workers. 670-676.

[57] Robbins, P. Stephen \& Judge, Timothy A. (2018). Organizational Behaviour, Edisi 13, Jilid 1, Salemba Empat, Jakarta.

[58] Sammy Godfrey Poro Sammy Godfrey Poro1, Andrew Peter Yiga, Julius Caesar Enon, Fabian Mwosi, Dr. Marus Eton. (2019). Teacher competence and performance in primary schools in Nwoya District, Northern Uganda. International Journal of Advanced Educational Research ISSN: 2455-6157; Impact Factor: RJIF 5.12. Volume 4; Issue 1;Page No. 03-08.

[59] Sarwat Sultan dan Muhammad Shafi (2014). Impact Of Perceived Teachers' Competence On Students' Performance: Evidence For Mediating/ Moderating Role Of Class Environment. i-manager's Journal o Psychology, Vol. No. 1.

[60] Sekaran, Uma dan Bougie, Roger. (2017). Metode Penelitian untuk Bisnis: Pendekatan keahlian. West Sussex: Wiley. Penerbit Salemba Empat.

[61] Shadma Absar dan Rasmita Das Swain. (2009). Organizational Commitment of University teachers across Discipline and Designation. Conference Paper · November 2009 DOI: 10.13140/2.1.4625.8246.

[62] Shamaki dan Timothy Ado (2015). Influence of Learning Environment on Students' Academic Achievement in Mathematics: A Case Study of Some Selected Secondary Schools in Yobe State - Nigeria. Journal of Education and Practice ISSN 2222-1735 (Paper) ISSN 2222-288X (Online) Vol.6, No.34, 2015.

[63] Siddique, Miss A., Hassan D Aslam, Mannan Khan, dan Unrooj Fatima (2011). Impact of Academic Leaderhip on Faculty'
Motivation, and Organizational Effectiveness in Higher Education System. International Journal of Business and Social Science, Vol. 2, No. 8, hal. 184-191.

[64] Simamora, Henry. (2012). Manajemen Sumber Daya Manusia. Edisi 1. Yogyakarta: STIE YKPN Yogyakarta.

[65] Spencer Jr, Lyle M., dan Signe M. Spencer (1993). Competence at Work: Models for Superior Performance. Edition. New Jersey: John Wiley \& Sons, Inc.

[66] Srikanthan, G. dan John Dalrymple. (2003). Developing Alternative Perspectives for Quality in Higher Education. The International Journal of Educational Management, Vol. 17, No. 2, hal. 126-136.

[67] Sugiyono. (2017). Metode Penelitian Kuantitatif, Kualitatif, dan $R$ danD. Bandung: Penerbit Alfabeta.

[68] Suharto, Suyanto, Nedi Hendri. (2018). The Impact of Organizational Commitment on Job Performance. International Journal of Economics and Business Administration Volume VII, Issue 2, 2019 pp. 189-206.

[69] Sukmadinata. (2009). Landasan Psikologi dan Proses Pendidikan. Bandung: PT Remaja Rosdakarya.

[70] Sumual, Moreen Zedko Isaura. Ali, Mohammad. (2017). Evaluation of Primary School Teachers' Pedagogical Competence in Implementing Curriculum. Journal of Education and Learning. Vol. 11 (3) pp. 343-350.

[71] Suryawan, K. A. G., Gani, A. J. A., \& Hanafi, I. (2020). The Role Of Leadership And Academic Atmosphere In Private Universities In Improving The Quality Of Lecturers: Study At Warmadewa University. International Research Journal Of Management, It And Social Sciences, 7(3), 72.

[72] Susanto, Ahmad. (2014). Teori Belajar dan Pembelajaran di Sekolah Dasar. Jakarta: Kencana Prenadamedia Group.

[73] Sutanto, E. M., Scheller-Sampson, J., \& Mulyono, F. (2018). Organizational Justice, Work Environment, And Motivation.

[74] Tarigan, N. M. R., \& Setiawan, H. (2020). The Effect Of Employee Competence On Increasing Employee Motivation In Sumut Bank Of Sharia Unit, North Sumatera. Budapest International Research And Critics Institute (Birci-Journal): Humanities And Social Sciences, 3(2), 858-867.

[75] Thrash, Alberta B. (2009). Leadership in Higher Education: an Analysis of the Leadership Styles of Academic Deans in Ohio's 13 State-Supported Universities. Dissertation. Capella University.

[76] Tugrul, Mart, Cagri. (2013). "The Audio-Lingual Method: An Easy way of Achieving Speech". International Journal of Academic Research in Business and Social Sciences. Vol. 3, No. 12.

[77] Utoyo, Bambang., Corry, Yohana dan Mardi. (2019).The Effect Of Competence And Organizational Culture, On Performance Of Employees With Work Motivation As MediationVariable. 33307828 .

[78] Veithzal, Rivai. 2015. Manajemen Sumber Daya Manusia Untuk Perusahaan. Jakarta: Rajawali Pers.

[79] Yanti, E. D., \& Sanny, A. (2019). The Influence Of Motivation, Organizational Commitment, And Organizational Culture To The Performance Of Employee Universitas Pembangunan Panca Budi. International Journal Of Research \& Review Vol, 6(10).

[80] Yekti Irawan, V. D., \& Usman, O. (2021). The Influence Of Parent's Attention, Reward Giving, And Learning Facilities On Students Learning Motivation. Ssrn Electronic Journal.

[81] Zacher, H., \& Rudolph, C. W. (2021). Relationships Between Psychological Contract Breach And Employee Well Being And Career Related Behavior: The Role Of Occupational Future Time Perspective. Journal Of Organizational Behavior, 42(1), 84-99.

[82] Zameer, H., Ali, S., Nisar, W., Amir, M., \& Sub Campus Layyah, B.-B. (2014). The Impact Of The Motivation On The Employee's Performance In Beverage Industry Of Pakistan. International Journal Of Academic Research In Accounting, 4(1), 293-298. 